\title{
Incidence of Hypoglycemia and Hospitalization Related to Chronic Diabetes Complications and Its Effect on Quality of Life Among Patients Initiating Second Line Therapy: DISCOVER Study
}

\author{
Khalid Al-Rubeaan ( $\nabla$ rubeaan@gmail.com ) \\ Research and Scientific Center, Sultan Bin Abdulaziz Humanitarian City, Riyadh \\ Faisal Banah \\ Armed Forces Hospital, Southern Region \\ Fayez Alruwaily \\ Prince Mutaib bin Abdul-Aziz Hospital \\ Eman Sheshah \\ King Salman bin Abdul-Aziz Hospital \\ Dhekra Alnaqeb \\ Department of internal medicine, Sultan Bin Abdulaziz Humanitarian City \\ Awad AlQahtani \\ Aseer Central Hospital \\ Diaa Ewais \\ Saudi German Hospital \\ Nassr Al Juhani \\ Al-Thager Hospital \\ Abdul-hameed Hassan \\ International Medical Center \\ Amira Youssef \\ Research and Scientific Center, Sultan Bin Abdulaziz Humanitarian City, Riyadh
}

\section{Research Article}

Keywords: DISCOVER study, hypoglycemia, hospitalization, HFS II, SF-36, microangiopathy, and cardiovascular events

Posted Date: December 10th, 2021

DOI: https://doi.org/10.21203/rs.3.rs-1102079/v1

License: @ (i) This work is licensed under a Creative Commons Attribution 4.0 International License. Read Full License 


\section{Abstract}

The management of patients with type 2 diabetes is a complex process that must be individualized and be patient centered. The aim of this study was to assess the metabolic control, the annual incidence and crude prevalence of hypoglycemia, hospitalization, and complications among patients with type 2 diabetes initiating second-line therapy. This study is an observational, longitudinal, prospective study as a part of the multinational DISCOVERing Treatment Reality of Type 2 Diabetes in Real World Settings (DISCOVER) study, that recruited 519 patients with type 2 diabetes who were non-insulin users, aged $\geq 18$ years, and switching to second-line therapy. The cohort was clinically evaluated over three years of follow up. Fear of hypoglycemia was assessed using the Hypoglycemia Fear Survey (HFS II), while the quality of life was assessed using SF36v2 questionnaire. Using second-line therapy improved metabolic control but the annual incidence of microangiopathies were at 61/1000 patient-years, 47/1000 patient-years, and 4/1000 patient-years for neuropathy, retinopathy, and nephropathy, respectively. The incidence of hypoglycemia was 57/1000 patient-years, where $50 \%$ were recurrent during the three-years period. The HFS II showed a significant increase in patients' worries related to hypoglycemia. The incidence of hospitalization was 31/1000 patient-years, out of which $8 / 1000$ patient-years were related to cardiovascular events, mainly myocardial infarction. Moderate metabolic control was associated with lower incidence of macro angiopathy and an increased incidence and fear of hypoglycemia, while it was associated with improved mental component score when assessing the patients' quality of life. The treating physician's decision of treatment intensification should be individualized with consideration of befits of good glycemic control versus the risk of hypoglycemia, especially in elderly patients.

\section{Introduction}

The management of patients with type 2 diabetes is a complex process that has to be individualized and to be patient centered. It usually follows clinical guidelines with consideration of the patient's personal and clinical characteristics. Patient's age, time since diabetes diagnosis, presence of comorbidities, risk of hypoglycemia, and the physician's experience are the most important factors that play a major role in the selection of management pathway. ${ }^{1}$ Type 2 diabetes is an established risk factor for cardiovascular disease, especially when associated with hypertension, obesity, and dyslipidemia, and plays an important role in the observed increasing mortality rates. ${ }^{2,3}$ The need for early achievement of good glycemic control to reduce the risk of microvascular and macrovascular complications among patients with type 2 diabetes was well established by the United Kingdom Prospective Diabetes Study (UKPDS) . ${ }^{\circ}$

New classes of glucose-lowering agents for treatment of type 2 diabetes are more commercially available globally in the last three decades. Despite new therapeutic classes, there is an increased risk of hospitalization, heart failure, myocardial infarction, and stroke. ${ }^{5}$ One explanation for such increased rates of complications is the association of good glycemic control with increased incidence of hypoglycemia which remains the main restricting factor for treatment intensification. ${ }^{6}$ Fear of hypoglycemia may also limit achieving good glycemic control and would have negative impact on the patients' quality of life. ${ }^{7}$

Healthcare providers and insurance companies are looking for data that predict unmet needs for the best approach to reach good control and reduced morbidity and morality that eventually reduce both direct and indirect cost.

\section{Objective}

As a part of the International multi-centered study (DISCOVER), ${ }^{8}$ which looks at patients with type 2 diabetes initiating second-line therapy, the objective of this study using the data collected from different centers from a country facing an epidemic of type 2 diabetes (Saudi Arabia) is to assess physicians, patients and health care system attitude towards patients failing first line management. Since it is a prospective study, it provides a better forecast for hospitalization related to chronic diabetes complications and hypoglycemic events in addition to the assessment of quality of life during the initiation of a second line therapy.

\section{Methods}

Study design and participants. The multinational DISCOVER study is an observational, longitudinal, prospective study conducted in nine hospitals in four out of the five provinces in Saudi Arabia. A total of 519 Saudi patients with type 2 diabetes, who were non-insulin users, aged $\geq 18$ years, and switching to secondline therapy were studied. The eligible patients were recruited between December 31st, 2014, and June 30th, 2016, to start a follow-up period of 36 months.

Eligible patients starting second-line therapy, either as an add-on or switching from first-line oral monotherapy, dual therapy, or triple therapy, were enrolled to participate in the study. Patients using an injectable agent, namely, insulin or a GLP-1-receptor agonist were excluded because of their disease severity. The exclusion criteria included patients with type 1 diabetes and pregnant women, patients using herbal remedies or natural medicines alone, and patients undergoing dialysis or with a history of renal transplantation.

Data collection. Eligible patients' data were collected using an electronic case report form (eCRF), and clinical evaluations including selected laboratory investigations at baseline and follow-ups at $6,12,24$, and 36 months were performed. Data were saved in a central database, and all eCRFs were checked for completeness by the study team.

During each visit, demographic and socioeconomic characteristics as well as physiological parameters, including blood pressure, pulse rate, weight, height, body mass index, and waist circumference, were collected. Any changes in glucose-lowering therapy reflected by an $\mathrm{HbA} 1 \mathrm{c}$ or fasting, random, or postprandial blood glucose were reported. Other laboratory parameters, including lipid parameters (total cholesterol, low-density lipoprotein (LDL), high-density lipoprotein (HDL), and triglycerides), renal function tests (serum creatinine and albumin/creatinine ratio), and uric acid were also collected. Liver function tests, including serum albumin, aspartate aminotransferase (AST), alanine aminotransferase (ALT), and gamma-glutamyl transpeptidase (GGT), were checked at each visit. The complete blood count parameters tested included white blood cells (WBC), hemoglobin (Hgb), hematocrit (Hct), and platelets. The investigators reported major and minor hypoglycemic events and comorbidities that involved microvascular and macrovascular complications. Microvascular complications were 
classified into nephropathy (chronic kidney disease and/or albuminuria), retinopathy (non-proliferative retinopathy (NPDR), proliferative retinopathy (PDR) or received retinal laser photocoagulation), and neuropathy (autonomic neuropathy, peripheral neuropathy, and/or erectile dysfunction). Macrovascular complications were coronary artery disease (angina, myocardial infarction, percutaneous coronary intervention, and coronary artery bypass grafting), heart failure, and implantable cardioverter defibrillator use. Cerebrovascular disease was reported in the form of stroke, transient ischemic attack (TIA), carotid artery stenting, or carotid endarterectomy. The peripheral artery diseases (PAD) reported included revascularization procedures, diabetic foot, and amputation. The major hypoglycemic events were defined as those that required an emergency room visit, hospital admission, a visit to a physician or other healthcare professional or external help from a caregiver or family member; and minor hypoglycemic events were defined as those which did not require external help.

The changes in HbA1c levels, body weight, blood pressure, and lipid profile were recorded in relation to treatment changes after the initiation of the second-line therapy or other glucose-lowering medications, insulin therapy, switching between glucose-lowering therapies, or dose changes. During the follow-up period, the incidence of microvascular complications, namely, nephropathy, neuropathy, and retinopathy was reported. The incidence of macrovascular complications was reported if the patient manifested heart failure, myocardial infarction, stroke, and diabetes-related revascularization. All patients' quality of life were assessed using the SF-36 questionnaire, and the fear of hypoglycemic events was assessed using the Hypoglycemia Fear Survey (HFS II), which assess the levels of fear related to hypoglycemia and consists of two subscales: Behavior (HFS-B) and Worry (HFS-W). ${ }^{9}$ Part of the data collected included incidence and reasons for hospitalizations or emergency room visits during the follow up period.

Statistical analysis. Descriptive analysis was used to describe demographic variables, patient characteristics, changes in $\mathrm{HbA1c}$ levels, blood glucose, lipid profile, body weight, body mass index, and blood pressure. The mean values were presented with standard deviations ( \pm SD) and the medians with interquartile ranges (IQR). All parameters were analyzed during the follow-up period using a P value of $<0.05$ as a level of significance. The per 1000 patient-years incidence of complications and hypoglycemic events either related or unrelated to diabetes were calculated. Frequency analyses were used to report clinical and demographic data for all participants and different subcategories. The categorical data are presented as numbers and percentages. Data from each domain of the Hypoglycemic Fear Survey (HFS II) were analyzed for descriptive statistics using the mean ( \pm SD) and the median (IQR). Multiple imputation was used to account for unreported data and missing values. Imputation was carried out by IVEware (University of Michigan). All statistical analyses were performed using the SAS statistical software system (SAS Institute, Inc., Cary, NC).

Ethical issues. The DISCOVER study protocol was approved by the relevant institutional review boards of the selected hospitals namely: the institutional review board, collage of medicine at King Saud university, General Administration for research and studies research ethics committee at Ministry of Health, the institutional review board at International Medical Center and the human research ethics committee of the Saudi German Hospital. All the study subjects signed informed consent forms. The protocol complies with the Declaration of Helsinki and the International Conference on Harmonization of Good Clinical Practice.

\section{Results}

A total of 477 patients completed the 36 months of follow-up visits and had clinical and biochemical assessments. Their anthropometric parameters did not show significant changes; even though waist circumference increased significantly, it may not carry any weight due to the non-significant change of mean body weight from the baseline. There was a significant reduction in all glycemic and lipid parameters in addition to diastolic blood pressure indicating better medical care. Serum creatinine and the albumin/creatinine ratio demonstrated a significant increase supporting the noted increase in the incidence of albuminuria of 22/1000 patient-years projecting the increase in the incidence of diabetic nephropathy as shown in Table 1. 
Table 1

Mean \pm SD and Median (IQR) for clinical, metabolic, and biochemical markers at baseline and during follow-up period

\begin{tabular}{|c|c|c|c|c|c|}
\hline \multirow[t]{3}{*}{ Parameter } & \multicolumn{5}{|l|}{ Follow-up Period } \\
\hline & Baseline & 6 months & 12 months & 24 months & 36 months \\
\hline & $(n=519)$ & $(n=470)$ & $(n=478)$ & $(n=473)$ & $(n=477)$ \\
\hline \multirow[t]{2}{*}{ Anthropometric parameters Weight (kg) } & $83.4 \pm 17.6$ & $83.3 \pm 17.6$ & $84.2 \pm 17.5$ & $82.7 \pm 16.9$ & $83.2 \pm 16.8$ \\
\hline & $82.0(71.0,93.0)$ & $81.0(71.0,93.0)$ & $83.0(72.0,94.0)$ & $81.0(71.0,91.0)$ & $82.0(70.0,92$. \\
\hline \multirow[t]{2}{*}{ Height (cm) } & $162.2 \pm 9.6$ & $162.0 \pm 9.3$ & $162.2 \pm 9.3$ & $161.7 \pm 9.0$ & $161.9 \pm 9.2$ \\
\hline & $162.0(155.0,170.0)$ & $161.0(155.0,169.0)$ & $162.0(155.0,169.0)$ & $160.5(155.0,168.0)$ & $161.0(155.0,1$ \\
\hline \multirow[t]{2}{*}{ BMI (m2/kg) } & $31.9 \pm 6.6$ & $31.9 \pm 6.7$ & $32.1 \pm 6.6$ & $32.0 \pm 6.6$ & $32.2 \pm 6.6$ \\
\hline & $31.1(27.5,35.3)$ & $31.1(27.3,35.6)$ & $31.5(27.8,35.6)$ & $31.5(27.4,35.1)$ & $32.0(27.7,35$. \\
\hline \multirow[t]{2}{*}{ Waist circumference (cm) } & $104.1 \pm 14.4$ & $103.2 \pm 14.1$ & $105.2 \pm 13.8$ & $104.1 \pm 12.5$ & $107.2 \pm 10.3$ \\
\hline & $104.0(95.0,112.0)$ & $103.0(96.0,111.0)$ & $104.0(97.0,114.0)$ & $103.0(96.0,110.5)$ & $108.0(105.0,1$ \\
\hline \multirow[t]{2}{*}{ Clinical paraments Pulse rate at rest (/min) } & $82.0 \pm 10.8$ & $80.7 \pm 10.2$ & $80.1 \pm 10.5$ & $79.8 \pm 10.3$ & $81.0 \pm 9.8$ \\
\hline & $81.0(75.0,88.0)$ & $80.0(74.0,88.0)$ & $80.0(72.0,87.0)$ & $80.0(72.0,86.0)$ & $80.0(76.0,87$. \\
\hline \multirow[t]{2}{*}{ Systolic BP (mmHg) } & $133.7 \pm 17.5$ & $132.4 \pm 15.8$ & $132.5 \pm 15.6$ & $132.0 \pm 16.2$ & $133.0 \pm 16.0$ \\
\hline & $132.0(122.0,143.0)$ & $131.5(120.0,141.0)$ & $133.0(120.0,142.0)$ & $130.0(120.0,140.0)$ & $130.0(120.0,1$ \\
\hline \multirow[t]{2}{*}{ Diastolic BP (mmHg) } & $79.2 \pm 10.9$ & $78.4 \pm 9.9$ & $78.0 \pm 10.1$ & $77.1 \pm 10.5$ & $77.5 \pm 9.8$ \\
\hline & $80.0(72.0,87.0)$ & $80.0(70.0,85.0)$ & $78.0(70.0,86.0)$ & $80.0(70.0,85.0)$ & $80.0(70.0,83$. \\
\hline \multirow[t]{3}{*}{ Metabolic markers $\mathrm{HbA} 1 \mathrm{C} \%$ (mmol/mol) } & $8.8 \pm 1.7(73)$ & $7.8 \pm 1.4(62)$ & $8.0 \pm 1.5(64)$ & $8.1 \pm 1.7(65)$ & $8.2 \pm 1.7(66)$ \\
\hline & $8.6(71)$ & $7.5(59)$ & $7.7(61)$ & $7.7(61)$ & $7.8(62)$ \\
\hline & $7.6(60), 9.8(84)$ & $6.8(51), 8.4(68)$ & $6.9(52), 8.8(73)$ & $6.9(52), 9.1(76)$ & $7.0(53), 9.1(7$ \\
\hline \multirow[t]{2}{*}{ Fasting Glucose (mg/dL) } & $185.9 \pm 65.5$ & $152.3 \pm 50.0$ & $154.6 \pm 50.0$ & $152.2 \pm 55.4$ & $146.3 \pm 55.1$ \\
\hline & $169.4(135.1,225.2)$ & $140.5(119.1,173.0)$ & $146.0(117.1,179.0)$ & $136.4(117.1,174.8)$ & $137.5(107.9,1$ \\
\hline \multirow[t]{2}{*}{ Random Glucose (mg/dL) } & $227.0 \pm 79.2$ & $199.1 \pm 67.2$ & $215.7 \pm 70.7$ & $241.1 \pm 86.4$ & $205.6 \pm 72.8$ \\
\hline & $215.3(173.0,278.0)$ & $191.0(151.0,225.2)$ & $203.5(167.6,251.0)$ & $234.2(183.0,298.0)$ & $192.5(155.0$, \& \\
\hline \multirow[t]{2}{*}{ Post Prandial Glucose (mg/dL) } & $256.2 \pm 78.5$ & $208.6 \pm 70.9$ & $197.0 \pm 63.5$ & $206.5 \pm 68.5$ & $167.3 \pm 31.0$ \\
\hline & $252.1(200.9,289.5)$ & $189.0(160.4,254.1)$ & $180.1(153.2,235.0)$ & $200.0(160.0,231.0)$ & $161.1(144.0,1$ \\
\hline \multirow[t]{2}{*}{ Lipid profile Total Cholesterol (mg/dL) } & $190.0 \pm 52.7$ & $175.8 \pm 40.8$ & $177.0 \pm 42.9$ & $174.6 \pm 35.6$ & $177.6 \pm 40.5$ \\
\hline & $184.0(160.2,210.0)$ & $169.9(150.0,196.0)$ & $170.3(150.6,199.6)$ & $172.2(152.9,197.7)$ & $176.0(151.4$, \& \\
\hline \multirow[t]{2}{*}{$\mathrm{HDL}(\mathrm{mg} / \mathrm{dL})$} & $44.0 \pm 11.5$ & $44.4 \pm 12.5$ & $44.3 \pm 11.1$ & $45.6 \pm 10.9$ & $47.0 \pm 11.0$ \\
\hline & $42.7(36.1,52.1)$ & $42.1(36.7,49.0)$ & $43.1(37.5,50.0)$ & $44.0(37.0,53.0)$ & $45.9(39.8,54$. \\
\hline \multirow[t]{2}{*}{ LDL (mg/dL) } & $112.0 \pm 35.7$ & $101.6 \pm 33.6$ & $103.2 \pm 33.2$ & $101.1 \pm 29.8$ & $106.2 \pm 35.8$ \\
\hline & $112.4(90.3,134.0)$ & $101.4(81.1,119.7)$ & $99.2(84.2,121.0)$ & $100.8(81.1,120.0)$ & $101.2(83.4,1:$ \\
\hline \multirow[t]{2}{*}{ Triglycerides (mg/dL) } & $190.7 \pm 160.5$ & $166.7 \pm 121.2$ & $172.0 \pm 115.9$ & $171.3 \pm 117.3$ & $153.7 \pm 82.9$ \\
\hline & $152.0(110.0,212.4)$ & $133.6(106.0,185.0)$ & $144.2(107.1,200.0)$ & $145.0(104.4,204.4)$ & $134.2(108.8,1$ \\
\hline \multirow{2}{*}{$\begin{array}{l}\text { Renal function Albumin/Creatinine ratio } \\
(\mathrm{mg} / \mathrm{g})\end{array}$} & $45.3 \pm 62.6$ & $28.8 \pm 44.3$ & $63.6 \pm 139.0$ & $89.9 \pm 252.6$ & $83.5 \pm 134.8$ \\
\hline & $18.4(8.0,59.0)$ & $8.4(5.9,34.0)$ & $9.8(6.4,31.7)$ & $15.2(2.9,34.5)$ & $37.7(9.0,62.0$ \\
\hline \multirow[t]{2}{*}{ Serum Creatinine } & $1.0 \pm 1.2$ & $1.2 \pm 1.5$ & $1.3 \pm 1.8$ & $2.1 \pm 2.7$ & $1.9 \pm 2.5$ \\
\hline & $0.8(0.7,1.0)$ & $0.8(0.7,1.0)$ & $0.8(0.7,1.0)$ & $0.9(0.7,1.2)$ & $0.8(0.7,1.2)$ \\
\hline \multirow[t]{2}{*}{ Uric acid (mg/dL) } & $4.7 \pm 1.3$ & $5.1 \pm 1.6$ & $5.1 \pm 1.5$ & $4.7 \pm 1.9$ & $4.8 \pm 1.2$ \\
\hline & $4.7(3.7,5.6)$ & $4.9(4.0,5.9)$ & $5.0(4.1,5.6)$ & $4.7(4.0,5.5)$ & $4.6(4.0,5.3)$ \\
\hline \multirow[t]{2}{*}{ Liver function Serum Albumin (g/dL) } & $4.2 \pm 0.4$ & $4.2 \pm 0.5$ & $4.2 \pm 0.7$ & $4.0 \pm 0.5$ & $4.1 \pm 0.4$ \\
\hline & $4.2(4.0,4.5)$ & $4.2(4.0,4.5)$ & $4.2(3.9,4.4)$ & $4.1(3.8,4.4)$ & $4.1(3.8,4.5)$ \\
\hline
\end{tabular}




\begin{tabular}{|c|c|c|c|c|c|}
\hline \multirow[t]{3}{*}{ Parameter } & \multicolumn{5}{|l|}{ Follow-up Period } \\
\hline & Baseline & 6 months & 12 months & 24 months & 36 months \\
\hline & $(n=519)$ & $(n=470)$ & $(n=478)$ & $(n=473)$ & $(n=477)$ \\
\hline \multirow[t]{2}{*}{ Alanine transaminase (ALT, IU/L) } & $34.1 \pm 21.9$ & $1.0 \pm 19.5$ & $29.9 \pm 15.7$ & $30.7 \pm 21.0$ & $31.6 \pm 18.4$ \\
\hline & $28.0(19.0,41.0)$ & $26.0(19.1,37.0)$ & $27.0(18.9,38.5)$ & $25.0(17.2,34.5)$ & $26.5(18.0,43$. \\
\hline \multirow[t]{2}{*}{ Aspartate transaminase (AST, IU/L) } & $22.1 \pm 13.0$ & $21.4 \pm 11.6$ & $23.0 \pm 18.0$ & $21.7 \pm 10.7$ & $21.1 \pm 9.2$ \\
\hline & $18.7(15.0,25.0)$ & $18.5(15.0,24.0)$ & $18.5(15.0,23.4)$ & $19.0(15.0,24.0)$ & $20.0(15.7,23$. \\
\hline \multirow[t]{2}{*}{ Gamma Glutamyltranspeptidase (GGT, IU/L) } & $46.2 \pm 40.1$ & $40.0 \pm 27.0$ & $48.1 \pm 45.5$ & $55.9 \pm 73.9$ & $45.2 \pm 95.3$ \\
\hline & $35.0(22.0,57.5)$ & $33.0(23.0,46.0)$ & $36.0(23.0,60.0)$ & $35.0(27.0,50.0)$ & $32.0(20.3,39$. \\
\hline \multirow[t]{2}{*}{ CBC White Blood Cell Count } & $8.1 \pm 2.3$ & $7.8 \pm 2.2$ & $8.3 \pm 2.5$ & $8.0 \pm 2.5$ & $7.8 \pm 3.3$ \\
\hline & $7.7(6.5,9.4)$ & $7.9(6.2,8.9)$ & $8.1(6.4,9.6)$ & $7.7(6.1,9.5)$ & $7.2(5.8,8.7)$ \\
\hline \multirow[t]{2}{*}{ Hemoglobin } & $13.5 \pm 1.7$ & $13.5 \pm 1.8$ & $13.1 \pm 2.0$ & $13.4 \pm 1.9$ & $13.8 \pm 1.6$ \\
\hline & $13.6(12.4,14.6)$ & $13.6(12.5,14.8)$ & $13.3(12.1,14.6)$ & $13.6(12.1,14.8)$ & $14.0(12.4,15$. \\
\hline \multirow[t]{2}{*}{ Hematocrit } & $41.6 \pm 5.0$ & $41.9 \pm 5.2$ & $41.8 \pm 5.2$ & $40.9 \pm 4.9$ & $41.8 \pm 4.6$ \\
\hline & $41.4(38.2,44.9)$ & $41.8(38.9,45.8)$ & $42.5(37.7,44.9)$ & $41.9(37.4,44.5)$ & $41.7(39.1,45$. \\
\hline \multirow[t]{2}{*}{ Platelets } & $268.0 \pm 72.7$ & $251.3 \pm 55.1$ & $268.4 \pm 75.4$ & $291.4 \pm 61.7$ & $264.1 \pm 79.1$ \\
\hline & $257.5(215.0,313.0)$ & $238.0(211.0,287.0)$ & $257.0(203.0,313.0)$ & $295.0(253.0,333.0)$ & $261.5(214.5,:$ \\
\hline
\end{tabular}

The incidence of mild hypoglycemia that did not warrant admission was 57/1000 patient-years, while the incidence of major hypoglycemic events was found to be $0.7 / 1000$ patient-years as shown in Table 2.

Table 2

Incidence and annual frequency of major and minor hypoglycemia during the three-year follow-up

\begin{tabular}{|c|c|c|c|c|c|c|}
\hline \multirow[t]{3}{*}{ Clinical condition } & & \multicolumn{4}{|c|}{ Follow-up period } & \multirow{3}{*}{$\begin{array}{l}\text { INCIDENCE } \\
\text { (/1000 patient- } \\
\text { years) }\end{array}$} \\
\hline & & \multirow{2}{*}{$\begin{array}{l}6 \text { months, } \\
\mathrm{n}=470 \\
\text { Number }(\%)\end{array}$} & \multirow{2}{*}{$\begin{array}{l}12 \text { months, } \\
\mathrm{n}=478 \\
\text { Number (\%) }\end{array}$} & \multirow{2}{*}{$\begin{array}{l}24 \text { months, } \\
n=473 \\
\text { Number (\%) }\end{array}$} & \multirow{2}{*}{$\begin{array}{l}36 \text { months, } \\
\mathrm{n}=477 \\
\text { Number (\%) }\end{array}$} & \\
\hline & & & & & & \\
\hline Major hypoglycemic event & & $1(0.2)$ & 0 & 0 & 0 & $1(0.7)$ \\
\hline Minor hypoglycemic event & & $21(4.8)$ & $15(3.2)$ & $19(4.0)$ & $26(5.5)$ & $81(57)$ \\
\hline \multirow{5}{*}{$\begin{array}{l}\text { Number of minor hypoglycemic attacks } \\
\text { in the previous } 4 \text { weeks }\end{array}$} & 1 & $5(1.1)$ & $6(1.3)$ & $8(1.7)$ & $11(2.3)$ & $30(21)$ \\
\hline & 2 & $7(1.5)$ & $4(0.8)$ & $8(1.7)$ & $7(1.5)$ & $24(17)$ \\
\hline & 3 & $7(1.5)$ & $1(0.2)$ & $3(0.6)$ & $2(0.4)$ & $13(9)$ \\
\hline & 4 & $1(0.2)$ & $3(0.6)$ & 0 & $1(0.2)$ & $5(3)$ \\
\hline & $\geq 5$ & $1(0.2)$ & $1(0.2)$ & 0 & $4(0.8)$ & $6(4)$ \\
\hline
\end{tabular}

The incidence of hospitalization was $31 / 1000$ patient-years, mainly as a single admission. Of those, $8 / 1000$ patient-years were related to cardiovascular events, 2/1000 patient-years were related to serious infection, and 1/1000 patient-years were related to cancer. While the incidence of emergency room visits was 81/1000 patient-years mainly single visits, as shown in Table 3 . The emergency room visits were mainly related to cardiovascular disease at an incidence of $10 / 1000$ patient-years, where $6 / 1000$ patient-years were due to myocardial infarctions and 4/1000 patient-years were related to heart failure that were mainly class II according to the New York Heart Association classification. 
Table 3

The crude prevalence and calculated incidence of hospitalizations and emergency visits during follow-up period for studied cohort

\begin{tabular}{|c|c|c|c|c|c|}
\hline \multirow[t]{3}{*}{ Medical care event } & \multicolumn{4}{|c|}{ Follow-up period } & \multirow{3}{*}{$\begin{array}{l}\text { INCIDENCE } \\
\text { (/1000 patient- } \\
\text { years) }\end{array}$} \\
\hline & \multirow{2}{*}{$\begin{array}{l}6 \text { months, } \\
\mathrm{n}=470 \\
\text { Number }(\%)\end{array}$} & \multirow{2}{*}{$\begin{array}{l}12 \text { months, } \\
\mathrm{n}=478 \\
\text { Number (\%) }\end{array}$} & \multirow{2}{*}{$\begin{array}{l}24 \text { months, } \\
\mathrm{n}=473 \\
\text { Number (\%) }\end{array}$} & \multirow{2}{*}{$\begin{array}{l}36 \text { months, } \\
\mathrm{n}=477 \\
\text { Number (\%) }\end{array}$} & \\
\hline & & & & & \\
\hline Hospitalizations since last follow-up & $8(1.7)$ & $8(1.7)$ & $12(2.5)$ & $16(3.4)$ & $44(31)$ \\
\hline \multirow{2}{*}{$\underset{1}{\text { Number of hospitalizations since last follow-up }}$} & $7(1.5)$ & $7(1.5)$ & $11(2.3)$ & $15(3.1)$ & $40(28)$ \\
\hline & $1(0.2)$ & $1(0.2)$ & $1(0.2)$ & $1(0.2)$ & $4(2.8)$ \\
\hline \multicolumn{6}{|l|}{2} \\
\hline Reason for hospitalization & $3(0.6)$ & $5(1.0)$ & $10(2.1)$ & $9(1.9)$ & 27 (19) \\
\hline Cardiovascular event & $4(0.9)$ & $2(0.4)$ & $1(0.2)$ & $5(1.0)$ & $12(8)$ \\
\hline Serious infections & 0 & $1(0.2)$ & $1(0.2)$ & $1(0.2)$ & $3(2)$ \\
\hline Cancer & $1(0.2)$ & 0 & 0 & $1(0.2)$ & $2(1.4)$ \\
\hline Renal failure & 0 & 0 & 0 & $1(0.2)$ & $1(0.7)$ \\
\hline Emergency room visits since last follow-up & $19(4.0)$ & $13(2.7)$ & $42(8.9)$ & $42(8.8)$ & $116(81)$ \\
\hline Number of emergency room visits 1 & $15(3.2)$ & $9(1.9)$ & $35(7.4)$ & $32(6.7)$ & $91(64)$ \\
\hline 2 & $3(0.6)$ & $3(0.6)$ & $5(1.1)$ & $8(1.7)$ & $19(13)$ \\
\hline$\geq 3$ & $1(0.2)$ & $1(0.2)$ & $2(0.4)$ & $2(0.4)$ & $5(3)$ \\
\hline $\begin{array}{l}\text { Reason for emergency room visits Cardiac } \\
\text { related }\end{array}$ & $2(0.4)$ & $2(0.4)$ & $5(1.1)$ & $6(1.3)$ & $15(10)$ \\
\hline Diabetes-related complication & $1(0.2)$ & $2(0.4)$ & $2(0.4)$ & $2(0.4)$ & $7(5)$ \\
\hline Neurologic related disorders & $1(0.2)$ & 0 & 0 & 0 & $1(0.7)$ \\
\hline Peripheral arterial disease related complication & 0 & 0 & 0 & $1(0.2)$ & $1(0.7)$ \\
\hline
\end{tabular}

The incidence of retinopathy was 47/1000 patient-years, mainly in the form of NPDR. The incidence of neuropathy was higher, at a rate of $61 / 1000$ patientyears, presenting mainly as erectile dysfunction. Hypertension and hyperlipidemia were found at an incidence of 52/1000 patient-years and $80 / 1000$ patientyears, respectively. Thyroid disorder events were found at an incidence rate of $67 / 1000$ patient-years, where $94 \%$ of the events were hypothyroidism. The incidence of cancer was found to be $4 / 1000$ patient-years, mainly colorectal and breast cancer. The most-commonly reported infection was urinary tract infection (UTI) with an incidence of 21/1000 patient-years. Depression was reported at an incidence of 8/1000 patient-years, as shown in Table 4 and appendix 1 . 
Table 4

The crude prevalence and calculated incidence of micro and macro angiopathy for studied cohort since previous follow-up visit.

\begin{tabular}{|c|c|c|c|c|c|}
\hline \multirow[t]{3}{*}{ Clinical condition } & \multicolumn{4}{|c|}{ Follow-up period } & \multirow{3}{*}{$\begin{array}{l}\text { INCIDENCE } \\
\text { (/1000 patient- } \\
\text { years) }\end{array}$} \\
\hline & $\begin{array}{l}6 \text { months, } \\
n=470\end{array}$ & $\begin{array}{l}12 \text { months, } \\
n=478\end{array}$ & $\begin{array}{l}24 \text { months, } \\
n=473\end{array}$ & $\begin{array}{l}36 \text { months, } \\
n=477\end{array}$ & \\
\hline & Number (\%) & Number (\%) & Number (\%) & Number (\%) & \\
\hline Cardiovascular event & $10(2.3)$ & $11(2.6)$ & $9(2.1)$ & $5(1.2)$ & $35(24)$ \\
\hline Myocardial infarction Events & $4(0.9)$ & $2(0.4)$ & $3(0.7)$ & $1(0.2)$ & $10(6)$ \\
\hline Single vessel disease & $4(0.9)$ & $2(0.4)$ & 0 & 0 & $6(4)$ \\
\hline Multiple vessels disease & 0 & 0 & $3(0.7)$ & $1(0.2)$ & $4(3)$ \\
\hline $\mathrm{PCl}$ & $2(0.5)$ & $3(0.7)$ & $4(0.9)$ & $2(0.5)$ & $11(8)$ \\
\hline $\mathrm{PCl}$ stent & $2(0.5)$ & $3(0.7)$ & $3(0.7)$ & $2(0.5)$ & $10(7)$ \\
\hline Other cardiac conditions Atrial & 0 & 0 & $1(0.2)$ & $2(0.5)$ & $3(2)$ \\
\hline Severe valve event & 0 & $1(0.2)$ & $1(0.2)$ & 0 & $2(1)$ \\
\hline Outpatient cardiac test & $14(3.3)$ & $8(1.9)$ & $4(0.9)$ & $5(1.2)$ & $31(22)$ \\
\hline $\begin{array}{l}\text { Heart failure according to NYHA class } \\
\text { Events }\end{array}$ & 0 & 0 & $4(0.9)$ & $2(0.5)$ & $6(4)$ \\
\hline (1) I & 0 & 0 & $1(0.2)$ & 0 & $1(0.7)$ \\
\hline (2) II & 0 & 0 & $2(0.4)$ & $2(0.4)$ & $4(3)$ \\
\hline (3) III & 0 & 0 & $1(0.2)$ & 0 & $1(0.7)$ \\
\hline Peripheral vascular disease Events & 0 & 0 & 0 & $2(0.5)$ & $2(1)$ \\
\hline PAD procedure & $1(0.2)$ & 0 & 0 & 0 & $1(0.7)$ \\
\hline DM foot event & $1(0.2)$ & $1(0.2)$ & 0 & 0 & $2(1)$ \\
\hline Chronic Kidney Disease Event & $1(0.2)$ & $1(0.2)$ & $1(0.2)$ & $4(0.9)$ & $7(4)$ \\
\hline Stage $2 \& 3$ eGFR $>30 \mathrm{~mL} / \mathrm{min} / 1.73 \mathrm{~m}^{2}$ & $1(0.2)$ & $1(0.2)$ & $1(0.2)$ & $2(0.4)$ & $5(1.0)$ \\
\hline Stage $4 \& 5$ eGFR $15-29 \mathrm{~mL} / \mathrm{min} / 1.73 \mathrm{~m}^{2}$ & 0 & 0 & 0 & $2(0.4)$ & $2(1.0)$ \\
\hline Albuminuria & $10(2.7)$ & $8(2.2)$ & $2(0.5)$ & $11(3.2)$ & $31(22)$ \\
\hline Retinopathy Events & $14(4.9)$ & $15(4.9)$ & $18(6.0)$ & $21(6.5)$ & $68(47)$ \\
\hline Maculopathy & 0 & $1(0.2)$ & $2(0.5)$ & $3(0.6)$ & $6(4)$ \\
\hline Non-proliferative & $13(2.8)$ & $14(2.9)$ & $12(2.5)$ & $17(3.6)$ & $56(39)$ \\
\hline Proliferative & $1(0.2)$ & 0 & $4(0.9)$ & $1(0.2)$ & $6(4)$ \\
\hline Peripheral neuropathy Events & $42(9.2)$ & $46(10.0)$ & $56(12.2)$ & $54(11.7)$ & $88(61)$ \\
\hline Autonomic neuropathy & $2(0.5)$ & $3(0.7)$ & $6(1.4)$ & $7(1.6)$ & $18(13)$ \\
\hline Erectile disfunction & $10(4.2)$ & $13(5.1)$ & $17(7.0)$ & $17(7.1)$ & $57(40)$ \\
\hline Outpatient neuropathy test & $2(0.5)$ & $4(0.9)$ & $1(0.2)$ & $6(1.4)$ & $13(9)$ \\
\hline Hypertension since last follow-up & $70(15.2)$ & $68(14.5)$ & $109(23.2)$ & $144(30.3)$ & $74(52)$ \\
\hline Hyperlipidemia since last follow-up & $63(14.5)$ & $63(14.4)$ & $135(29.4)$ & $177(38.0)$ & $114(80)$ \\
\hline Thyroid Disease Events & $21(4.8)$ & $17(3.8)$ & $28(6.1)$ & $30(6.4)$ & $96(67)$ \\
\hline Hyperthyroidism & 0 & 0 & $1(0.2)$ & $3(0.6)$ & $4(3)$ \\
\hline Hypothyroidism & $21(4.8)$ & $16(3.3)$ & $27(5.7)$ & $26(5.5)$ & $90(63)$ \\
\hline Arthritis event & $5(1.1)$ & $9(2.0)$ & $11(2.4)$ & $10(2.1)$ & $35(24)$ \\
\hline Cancer Events & $1(0.2)$ & $1(0.2)$ & $2(0.5)$ & $2(0.5)$ & $6(4)$ \\
\hline
\end{tabular}




\begin{tabular}{|c|c|c|c|c|c|}
\hline \multirow[t]{3}{*}{ Clinical condition } & \multicolumn{4}{|c|}{ Follow-up period } & \multirow{3}{*}{$\begin{array}{l}\text { INCIDENCE } \\
\text { (/1000 patient- } \\
\text { years) }\end{array}$} \\
\hline & $\begin{array}{l}6 \text { months, } \\
n=470\end{array}$ & $\begin{array}{l}12 \text { months, } \\
n=478\end{array}$ & $\begin{array}{l}24 \text { months, } \\
n=473\end{array}$ & $\begin{array}{l}36 \text { months, } \\
\mathrm{n}=477\end{array}$ & \\
\hline & Number (\%) & Number (\%) & Number (\%) & Number (\%) & \\
\hline Cancer site Breast & 0 & 0 & $1(0.2)$ & 0 & $1(0.7)$ \\
\hline Colorectal & 0 & $1(0.2)$ & $1(0.2)$ & 0 & $2(1)$ \\
\hline Other & $1(0.20)$ & 0 & 0 & $2(0.5)$ & $3(2)$ \\
\hline Urinary tract infection Events & $10(2.2)$ & $3(0.6)$ & $7(1.5)$ & $10(2.1)$ & $30(21)$ \\
\hline Genital infection events & $2(0.4)$ & 0 & $4(0.9)$ & $3(0.6)$ & $9(6)$ \\
\hline Respiratory disease & $2(0.5)$ & $3(0.7)$ & $4(0.9)$ & $9(2.1)$ & $18(13)$ \\
\hline Depression event & $1(0.2)$ & $2(0.5)$ & $3(0.7)$ & $5(1.1)$ & $11(8)$ \\
\hline
\end{tabular}

The Hypoglycemia Fear Survey Score (HFS II) for the total cohort demonstrated a significant increase in both behavior and worries mean and median scores throughout the three years of follow up, whereas the behavior mean score increased from $7.2 \pm 11.6$ at baseline to $8.2 \pm 12.1$ at 36 months follow up with a $p$ value 0 f 0.0238 , while worries means increased from $6.4 \pm 11.9$ at baseline to $7.9 \pm 11.6$ at 36 months follow up with a $p$ value of 0.0446 as shown in figure 1 .

Table 5 demonstrates the physical and mental components scores for the quality of life for the study participants through the follow up period. The mental component scores were significantly higher in each follow up visit compared with the baseline $(p<0.0001)$ with the highest mean score in the 24 months visit at $53.8 \pm 7.1$. The score differences from baseline were 4.0, 6.40 and 5.6 respectively. However, the mean physical component score did not change significantly during the 36 months follow up period

Table 5

The mean physical and mental component) scores of 36-item Short Form Health Survey version 2 (SF-36v2) from baseline to 36 months follow up.

\begin{tabular}{|c|c|c|c|c|c|}
\hline \multirow[t]{2}{*}{ SF36v2 measures } & \multicolumn{5}{|l|}{ Follow up period } \\
\hline & $\begin{array}{l}\text { Baseline } \\
(n=519)\end{array}$ & $\begin{array}{l}6 \text { months } \\
(n=470)\end{array}$ & $\begin{array}{l}12 \text { months } \\
(n=478)\end{array}$ & $\begin{array}{l}24 \text { months } \\
(\mathrm{n}=473)\end{array}$ & $\begin{array}{l}36 \text { months } \\
(\mathrm{n}=477)\end{array}$ \\
\hline SF-36 v2 Physical Component Score & $51.0 \pm 7.0$ & $49.0 \pm 7.0$ & $49.2 \pm 6.2$ & $50.4 \pm 5.7$ & $49.8 \pm 5.3$ \\
\hline $\begin{array}{l}\text { SF-36 v2 } \\
\text { Physical Component Score (Median (IQR)) }\end{array}$ & $51.9(47.0,56.0)$ & $50.4(43.2,54.2)$ & $49.3(44.2,54.2)$ & $51.0(46.0,54.9)$ & $50.6(45.9,54.1)$ \\
\hline SF-36 v2 Mental Component Score & $47.4 \pm 9.1$ & $51.4 \pm 8.0$ & $52.8 \pm 8.4$ & $53.8 \pm 7.1$ & $53.0 \pm 6.7$ \\
\hline SF-36 v2 Mental Component Score & $48.6(40.9,54.4)$ & $52.5(47.3,57.4)$ & $53.7(48.5,59.4)$ & $54.8(49.5,58.5)$ & $53.7(49.1,57.1)$ \\
\hline
\end{tabular}

\section{Discussion}

In this study, the second-line management had significantly improved the metabolic control of diabetes in the form of reducing $\mathrm{HbA} 1 \mathrm{c}$ during the 36 months follow up period. These real-world findings were consistent with a recent meta-analysis in which all investigated drug classes lowered $\mathrm{HbA1c}$ to a similar extent. ${ }^{10}$ The second-line treatments chosen for glycemic control by the treating physicians were not associated with risk of weight gain or significant severe hypoglycemia as observed in other studies. ${ }^{10}$ The physicians' attitude of clinical inertia observed in this study had affected the degree of reduction in glycemic control to reach the targeted level which is commonly observed in similar studies in different societies ${ }^{11}$. Another explanation could be related to the delay in introducing the second -line therapy which was observed in IMPROVE study that was conducted in eight countries and involved more than 50,000 patients which highlighted the concern that the initiation of second line therapy, particularly insulin, is commonly delayed in clinical practice. ${ }^{12}$

The improvement in glycemic parameters with the add on of the second-line therapy was reflected on the lipid parameters, with a significant decrease in the total cholesterol, LDL, and triglycerides, and an increase in HDL. It was reflected by reduced crude prevalence of cardiovascular disease during the follow up period especially at the first six months of follow up (2.3\%). Such observation was more pronounced in the Western countries but observed at a lower extent in the South-East Asian countries. ${ }^{13}$ The reduction in the crude prevalence of cardiovascular disease in this study could be the result of both mild improvement of glycemic control and lower mean age of this cohort. This highlights the importance of age, as a risk for cardiovascular disease which could outmost

Page $8 / 11$ 
glycemic control in this community. The same finding was also observed among patients with ischemic stroke in this community. ${ }^{14}$ Such observation was noted in patients with PAD manifested by foot ischemia at an incidence rate of $0.2 \%$, that was lower than what has been reported from the same study in Europe at a rate of $1.2 \%,{ }^{13}$ which could be a reflection of the younger age of our study cohort and in addition to ethnicity effect.

This cohort also demonstrated an increase in the crude prevalence of microvascular complications, namely: neuropathy, retinopathy, and nephropathy. Each year, for every 1000 patients with type 2 diabetes, 61 will present with neuropathy, 47 with retinopathy, and 4 with chronic renal disease. This increase in the crude prevalence could be explained by the mild reduction in $\mathrm{HbA1c}$ that did not meet the recommended targes and the legacy effect in the early phases of diabetes management ${ }^{15}$ which warrants more therapeutic intensification and earlier interventions. ${ }^{16}$

The all-cause hospitalization rate was ten times lower than what was reported in the General Practice Research Database linked to the Hospital Episode Statistics data in England, when for diabetes type and mean age. ${ }^{17}$ This could be explained by the limited access to in-patients' admission because of long waiting list. The second reason behind such low hospitalization rate could reflect low incidence of sever complications that warrants admission. In addition, with optimization of diabetes management in the study cohort, hypoglycemia was reported to be more limited to minor events with low recurrence rate that did not warrant hospitalization or emergency room visits.

The main reason for admission in this cohort was cardiovascular disease, which is in accordance with what has been reported in the global DISCOVER data. ${ }^{13}$ cardiovascular disease was also the most frequent reason for emergency room visit as expected in such patients. ${ }^{18}$ This study confirmed the fact that patients with diabetes are more prone to associated disease like thyroid disease, osteoarthritis, urinary and chest infections. Such findings support the fact that diabetes mellitus is associated with increased direct and indirect cost due to its complications and associated diseases which pressurize health services and economy. ${ }^{19}$

The fear of hypoglycemia restricts patients' likelihood of achieving and maintaining glycemic control and has a negative impact on their quality of life. ${ }^{20}$ The HFS II worry score was associated with the use of first-line insulin secretagogues and the glycemic response to second-line agents or insulin use in the studied cohort. The score for behavior, worries, or both significantly increased with more interventions or loss of glycemic control, which is the same finding reported from the same study at an international level by Wang et al.,. ${ }^{21}$ This finding underlines the importance of assessing patients' fear of hypoglycemia prior to treatment intensification, especially if comorbidities are present. On the other hand, the treatment intensification, and the add-on of the second line therapy was associated with improved quality of life (QoL) in terms of mental component score. The difference between the mean QOL score at the baseline and at any follow up visit exceeded the minimal clinically important difference for SF-36v2 score which is more than 3 points. ${ }^{22}$ There was an improvement in the mental QoL more than the physical QoL with tight glycemic control which is in line with finding of Lau et.al.,. ${ }^{23} \mathrm{~A}$ possible explanation of such finding is that increased regimen complexity required to achieve better glycemic control and increased risk of hypoglycemia may have a negative impact on patients' perception of physical QOL. On the other hand, increased sense of empowerment associated with improved glycemic control positively impacted the mental component of QOL. ${ }^{24}$

The improvement of quality of life despite the increased fear of hypoglycemia indicates that the patients' quality of life is affected more with the improvement of their glycemic control compared with the hypoglycemic fear.

Study limitations. This study has the strength of being a prospective longitudinal hospital-based and being part of an international multicenter study with low dropout rate of $8.1 \%$. The study limitations are related to data collection from the medical file that might have missing data like $\mathrm{HbA} 1 \mathrm{c}$, microalbuminuria, neuropathy testing, and fundus examination. Another limitation is the assumption of patients' adherence to their medications.

\section{Conclusions}

In conclusion, early initiation of second-line management would improve glycemic control and reduce complications rate, especially if the targeted level for both blood sugar and HbA1c is achieved. Hypoglycemia should not be a restricting factor when initiating second-line therapy, especially with better patients' education and closed home glucose monitoring. Improved diabetes control was associated with improved quality of life in terms of the mental and physical component scores. The treating physicians should individualize their plan for treatment intensification and assess the befits of good glycemic control versus the risk of hypoglycemia, especially in elderly patients.

Despite the low incidence rate of diabetes complications, this rate is still critical for a country facing type 2 diabetes epidemic, especially when no active prevention program has been adopted or lunched. The annual incidence of these complications could affect the health care. The study findings are baseline for health planners and insurance companies to improve health care and reduce the disease financial impact..

\section{Declarations}

\section{Acknowledgments}

We acknowledge the support given by staff in different participating hospitals and the staff of the research and scientific center at Sultan Bin Abdulaziz Humanitarian City for the support given in writing up this manuscript.

\section{Funding}

This study was funded by the AstraZeneca Company. 


\section{Conflict of Interest}

The authors declare that they have no competing interests.

\section{Data Availability}

The data that support the findings of this study are available from the DISCOVER study database, but restrictions apply to the availability of these data, which were used under license for the current study, and so are not publicly available.

\section{Author contribution}

Concept - K.A.; Design - K.A.; Supervision - K.A.; Data Collection and/or Processing - K.A.; Analysis and/or Interpretation - K.A., A.Y.; Writing - K.A., A.Y.;

Critical Reviews - K.A., F.A., E.S., D.A., A.A., N.A., A.H., D.E., A.Y.

\section{Disclosure}

All authors declared no competing interest. All views, scientific finding, conclusions, and recommendations mentioned in the study represent the sole opinion of the research team and do not in any way reflect the sponsoring company views.

\section{References}

1. Inzucchi, S.E. et al. Management of hyperglycemia in type 2 diabetes, 2015: a patient-centred approach. Update to a position statement of the American Diabetes Association and the European Association for the Study of Diabetes. Diabetologia. 58, 429-42 (2015).

2. Preis, S.R. et al. Trends in cardiovascular disease risk factors in individuals with and without diabetes mellitus in the Framingham heart study. Circulation $120,212-20$ (2009)

3. Al-Rubeaan, K.A. et al. All-cause Mortality and Its Risk Factors Among Type 1 and Type 2 Diabetes Mellitus in a Country Facing Diabetes Epidemic. Diabetes Res Clin Pract. 118, 130-9 (2016)

4. UK Prospective Diabetes Study (UKPDS) Group. k. Lancet 352,837-53 (1998).

5. Marso, S.P. et al. Liraglutide and cardiovascular outcomes in type 2 diabetes. N Engl J Med. 375, 311-22 (2016).

6. Cryer, P.E. Hypoglycemia: still the limiting factor in the glycemic management of diabetes. Endocr Pract. 2008 Sep;14(6):750-6. doi: 10.4158/EP.14.6.750. PMID: 18996798.

7. Amiel, S.A., Dixon, T., Mann, R. \& Jameson, K. Hypoglycaemia in Type 2 diabetes. Diabet Med. 25, 245-54 (2008).

8. Marilia, B. et al. Treatment of type 2 diabetes mellitus worldwide: Baseline patient characteristics in the global DISCOVER study, diabetes research and clinical practice 151,20-32 (2019).

9. Amanda Yun Rui Lam, Xiaohui Xin, Wee Boon Tan, Daphne Su-Lyn Gardner, Su-Yen Goh; Psychometric validation of the Hypoglycemia Fear Survey-II (HFS-II) in Singapore, BMJ Open Diab Res Care. 5, e000329 (2017).

10. Palmer SC, Mavridis D, Nicolucci A, et al. Comparison of clinical outcomes and adverse events associated with glucose-lowering drugs in patients with type 2 diabetes: a meta-analysis. JAMA. 2016; 316:313-324. doi: 10.1001/jama.2016.9400.

11. Khunti, S., Davies, M.J., \& Khunti, K. Clinical inertia in the management of type 2 diabetes mellitus: a focused literature review. The British Journal of Diabetes \& Vascular Disease 15, 65-69 (2015).

12. Valensi, P. et al. IMPROVE Study Group Expert Panel. The IMPROVE study-a multinational, observational study in type 2 diabetes: baseline characteristics from eight national cohorts. Int J Clin Pract. 62,1809-19 (2008).

13. Kosiborod, M. et al. DISCOVER investigators. Vascular complications in patients with type 2 diabetes: prevalence and associated factors in 38 countries (the DISCOVER study program). Cardiovasc Diabetol.17,150 (2018).

14. Al-Rubeaan, K., Al-Hussain, F., Youssef A.M., Subhani, S.N., Al-Sharqawi, A.H. \& Ibrahim, H.M. Ischemic Stroke and Its Risk Factors in a Registry-Based Large Cross-Sectional Diabetic Cohort in a Country Facing a Diabetes Epidemic. J Diabetes Res. 2016, 4132589 (2016).

15. Laiteerapong N, Ham SA, Gao Y, et al. The Legacy Effect in Type 2 Diabetes: Impact of Early Glycemic Control on Future Complications (The Diabetes \& Aging Study). Diabetes Care. 42, 416-426 (2019).

16. Folli, F. et al. The Role of Oxidative Stress in the Pathogenesis of Type 2 Diabetes Mellitus Micro- and Macrovascular Complications: Avenues for a Mechanistic-Based Therapeutic Approach. Curr Diabetes Rev.7,313-24 (2011).

17. Khalid, J. M. et al. Rates and risk of hospitalisation among patients with type 2 diabetes: retrospective cohort study using the UK General Practice Research Database linked to English Hospital Episode Statistics. Int J Clin Pract. 68,:40-8 (2014).

18. Morena Ustulin, Junghoon Woo, Jeong-taek Woo, and Sang Youl Rhee; Characteristics of frequent emergency department users with type 2 diabetes mellitus in Korea; J Diabetes Investig. 9, 430-437 (2018)

19. Care D. Economic Costs of Diabetes in the US in 2017. Diabetes Care. 41,917 (2018).

20. Barendse, S., Singh, H., Frier, B.M. \& Speight, J. The impact of hypoglycaemia on quality of life and related patient-reported outcomes in type 2 diabetes: a narrative review. Diabet Med. 29, 293-302 (2012).

21. Jun-Sing, W. Hungta, C. Fengming, T. \& Wayne Huey-Herng, S. Associations of fear of hypoglycemia with second-line use of insulin secretagogues or insulin and subsequent glycemic control in patients with type 2 diabetes: An analysis using data from the DISCOVER study. Int J Clin Pract. 31 , e13485 
(2020).

22. Frendl, D.M. \& Ware Jr, J.E. Patient-reported functional health and well-being outcomes with drug therapy: a systematic review of randomized trials using the SF-36 health survey. Med Care. 52, 439-45 (2014).

23. Lau, C.Y., Qureshi, A.K. \& Scott, S.G. Association between glycemic control and quality of life in diabetes mellitus. J Postgrad Med. 50,189-95 (2004).

24. Nomura, M., Fujimoto, K., Higashino, A., Denzumi, M., Miyagawa, M., Miyajima H, et al. Stress and coping behavior in patients with diabetes mellitus. Acta Diabetol. 37, 61-4(2000).

\section{Figures}

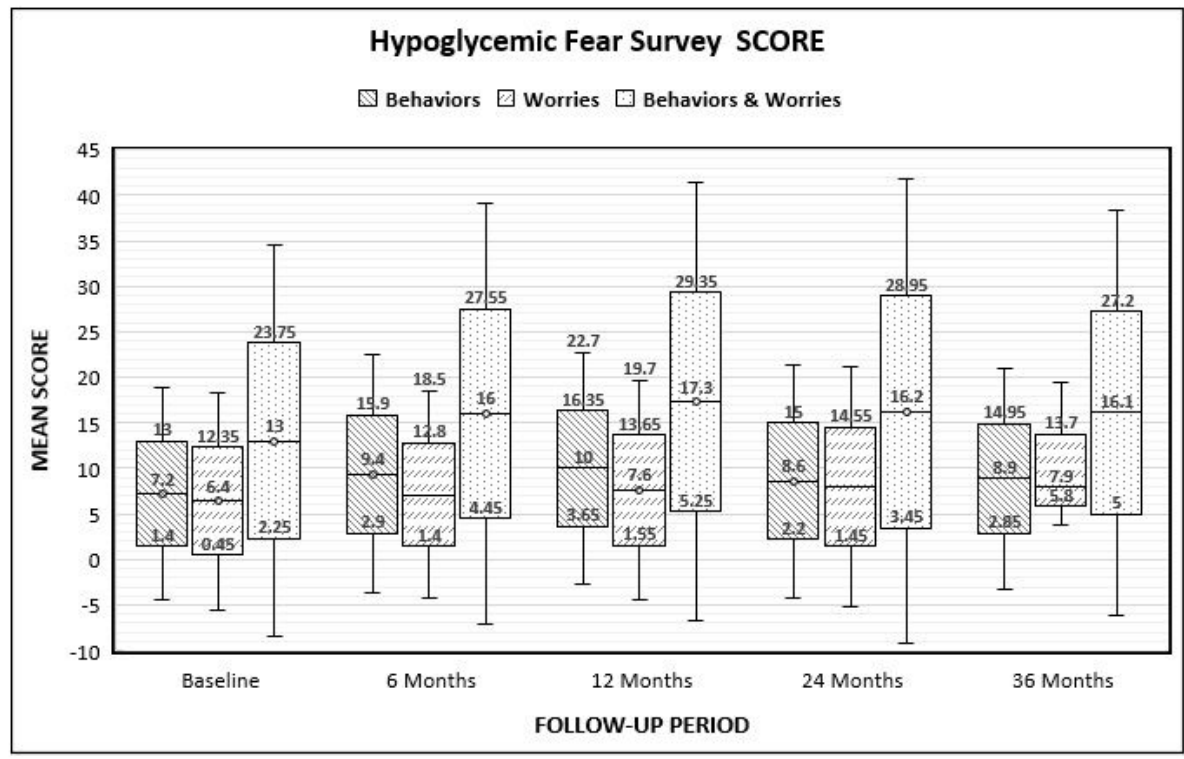

\begin{tabular}{|c|c|c|c|c|c|}
\hline $\begin{array}{c}\text { Hypoglycemic Fear Survey Score } \\
\text { (HFS II) }\end{array}$ & $\begin{array}{c}\text { Baseline } \\
\text { Number (519) }\end{array}$ & $\begin{array}{c}6 \text { months } \\
\text { Number }(470)\end{array}$ & $\begin{array}{c}12 \text { months } \\
\text { Number (478) }\end{array}$ & $\begin{array}{c}24 \text { months } \\
\text { Number (473) }\end{array}$ & $\begin{array}{c}36 \text { months } \\
\text { Number (477) }\end{array}$ \\
\hline Behaviors: mean ( $(\mathrm{SD})$ & $7.2 \pm 11.6$ & $9.4 \pm 13.0$ & $10.0 \pm 12.7$ & $8.6 \pm 12.8$ & $8.9 \pm 12.1$ \\
\hline$P$ value related to baseline & 1.0000 & 0.0050 & 0.0003 & 0.0710 & 0.0238 \\
\hline Behaviors: median (IQR) & $0.0(0.0,11.0)$ & $2.0(0.0,17.0)$ & $4.0(0.0,17.0)$ & $0.0(0.0,15.0)$ & $2.0(0.0,18.0)$ \\
\hline Worries: mean $( \pm S D)$ & $6.4 \pm 11.9$ & $7.1 \pm 11.4$ & $7.6 \pm 12.1$ & $8.0 \pm 13.1$ & $7.9 \pm 11.6$ \\
\hline$P$ value related to baseline & 1.0000 & 0.3462 & 0.1149 & 0.0441 & 0.0446 \\
\hline Worries: median (IQR) & $0.0(0.0,7.0)$ & $0.0(0.0,12.0)$ & $0.0(0.0,10.0)$ & $0.0(0.0,12.0)$ & $0.0(0.0,14.0)$ \\
\hline Behaviors and worry: mean ( \pm SD) & $13.0 \pm 21.5$ & $16.0 \pm 23.1$ & $17.3 \pm 24.1$ & $16.2 \pm 25.5$ & $16.1 \pm 22.2$ \\
\hline$P$ value related to baseline & 1.0000 & 0.0347 & 0.0030 & 0.0324 & 0.0254 \\
\hline Behaviors and worry: median (IOR) & $0.0(0.0,17.0)$ & $3.0(0.0,25.0)$ & $4.5(0.0,27.0)$ & $0(0.0,19.0)$ & $0(0.0,33.0)$ \\
\hline
\end{tabular}

\section{Figure 1}

Hypoglycemic Fear Survey scores at baseline and during follow-up period

\section{Supplementary Files}

This is a list of supplementary files associated with this preprint. Click to download.

- Appendix1.docx 